\title{
Multiple shRNA combinations for near-complete coverage of all HIV-1 strains
}

\author{
Glen J Mcintyre*, Jennifer L Groneman, Yi-Hsin Yu, Anna Tran, Tanya L Applegate
}

\begin{abstract}
Background: Combinatorial RNA interference (co-RNAi) approaches are needed to account for viral variability in treating HIV-1 with RNAi, as single short hairpin RNAs (shRNA) are rapidly rendered ineffective by resistant strains. Current work suggests that 4 simultaneously expressed shRNAs may prevent the emergence of resistant strains.

Results: In this study we assembled combinations of highly-conserved shRNAs to target as many HIV-1 strains as possible. We analyzed intersecting conservations of 10 shRNAs to find combinations with 4+ matching the maximum number of strains using 1220+ HIV-1 sequences from the Los Alamos National Laboratory (LANL). We built 26 combinations of 2 to 7 shRNAs with up to $87 \%$ coverage for all known strains and $100 \%$ coverage of clade B subtypes, and characterized their intrinsic suppressive activities in transient expression assays. We found that all combinations had high combined suppressive activities, though there were also large changes in the individual activities of the component shRNAs in our multiple expression cassette configurations.

Conclusion: By considering the intersecting conservations of shRNA combinations we have shown that it is possible to assemble combinations of 6 and 7 highly active, highly conserved shRNAs such that there is always at least 4 shRNAs within each combination covering all currently known variants of entire HIV-1 subtypes. By extension, it may be possible to combine several combinations for complete global coverage of HIV-1 variants.
\end{abstract}

\section{Introduction}

HIV is characterized by high sequence variability with many hundreds of genetically unique strains $[1,2]$. These are classified based on changes in the viral envelope with 3 groups ( $\mathrm{M}, \mathrm{N}$, and $\mathrm{O}$ ) and several subtypes (or clades). There is a geographical clustering for each, with group $M$ the main grouping globally and clade $B$ the most common subtype in USA and Europe [2]. The only effective way to currently treat HIV is with the simultaneous use of multiple antiretroviral drugs to prevent the emergence of drug-resistant strains [3]. RNA interference (RNAi) is a recently discovered mechanism of gene suppression that has received considerable attention for its potential use in gene therapy strategies for HIV (for review see [4-6]). Expressed short hairpin RNA (shRNA) effectors are well suited for potential use in gene therapy. Sharing structural similarities to natural microRNA, shRNA consists of a short single stranded RNA transcript that folds into a 'hairpin' configuration

\footnotetext{
* Correspondence: glen@madebyglen.com

Johnson and Johnson Research Pty Ltd, Level 4 Biomedical Building, 1 Central Avenue, Australian Technology Park, Eveleigh, NSW, 1430, Australia
}

by virtue of self-complementary regions separated by a short 'loop' sequence. shRNA is commonly expressed from U6 and H1 pol III promoters. These promoters are compact, active in many tissues, and are well suited to shRNA expression due to their relatively well-defined transcription start and end points. Importantly, pol III based shRNA expression cassettes have been incorporated into viral vectors which have been stably integrated both in culture and whole animals with effective silencing maintained over time [7-9].

The potency of individual shRNA has been extensively demonstrated in culture and there are now several hundred identified targets and verified shRNAs for HIV [10-12]. However, single shRNAs can be rapidly overcome by viral escape mutants possessing small sequence changes that alter the structure or sequence of the targeted region [13-16]. A combinatorial RNAi approach using multiple shRNAs is required to prevent the emergence of resistant strains [17-19], with models predicting that as few as 4 shRNAs will be sufficient $[19,20]$. However, this requires all 4 shRNAs to be matched to each of the 100's of circulating viral variants spanning the
C Biomed Central

() 2011 Mcintyre et al; licensee BioMed Central Ltd. This is an Open Access article distributed under the terms of the Creative Commons Attribution License (http://creativecommons.org/licenses/by/2.0), which permits unrestricted use, distribution, and reproduction in any medium, provided the original work is properly cited. 
different subtypes. Previously reported combinations have shown much promise in laboratory tests, though a number are of limited clinical relevance in terms of target sequences $[11,16,21-23]$. This is because they tend to be assembled on the basis of the individual conservations of the component shRNAs without consideration of the intersecting conservation of the entire combination, where the highest individual conservations are not necessarily reflected in the intersecting conservation. This is an important point, as some strains will be (inadequately) covered by fewer than the intended number of shRNAs, thus facilitating the emergence of escape mutants. Unless all 4 shRNAs are effectively conserved across all targeted strains (unlikely), then more than 4 shRNAs will be required to attain at least 4 matched to each different strain (Figure 1).

There are a number of potential methods for coexpressing multiple shRNA, including: multiple expression vectors $[9,24,25]$, multiple expression cassettes from a single vector $[11,26,27]$, and long single transcripts composed of an array of multiple shRNA domains $[16,21,28-30]$. The latter strategy is advantageous for gene therapy as it uses the fewest promoters, is the most compact and can be designed to mimic natural polycistronic miRNA clusters [22,30-32]. However, it is also the most difficult to currently use with many design variations and no clear guidelines. We and others have found that the original suppressive activities of the component shRNAs were not necessarily maintained in combination, and combinations of more than 2 shRNAs became increasingly difficult to assemble [16,28,29,33]. Moreover, effective combinations may be limited to only 3 domains, which is too few [34]. The multiple cassette strategy is a most useful method for immediate use due to its ease of design, assembly, and direct compatibility with pre-existing active shRNA. Others have also used this co-expression strategy to investigate multiple shRNA treatments for viral diseases, using cassette combinations ranging from 2 to $6[11,26,27,35,36]$.

The primary aim of this study was to mathematically assemble and select combinations of highly-conserved anti-HIV shRNAs to target a maximum number of viral variants whilst minimizing the risk of selecting for escape mutants. We also aimed to characterize the intrinsic individual and combined suppressive activities of the component shRNAs when simultaneously expressed. We made 26 combinations of 2 to 7 shRNA with some containing at least 4 shRNA fully matched to $100 \%$ of clade B sequences, and up to $87 \%$ of all other clades. We found that while all combinations had high combined suppressive activities, the individual activities of the component shRNAs could vary compared to the corresponding single shRNAs. Importantly, we present a method by which highly relevant combinations can be selected, and have shown that a surprisingly small number of shRNAs can combined into single combinations with the potential for targeting entire subtype groups.

\section{Results}

\section{A selection of anti-HIV shRNA}

We have previously analyzed over 8000 unique 19 nucleotide (nt.) HIV-1 targets, and calculated their level of conservation amongst almost $38000 \mathrm{HIV}$ gene sequence fragments containing 24.8 million 19 mers [12]. We characterized 96 in detail, and 10 of these, spanning 7 genes, were selected for assembly into combinations here $(\# 0$ - 9) (Table 1). This selection was based on a combination of activity, conservation and
A

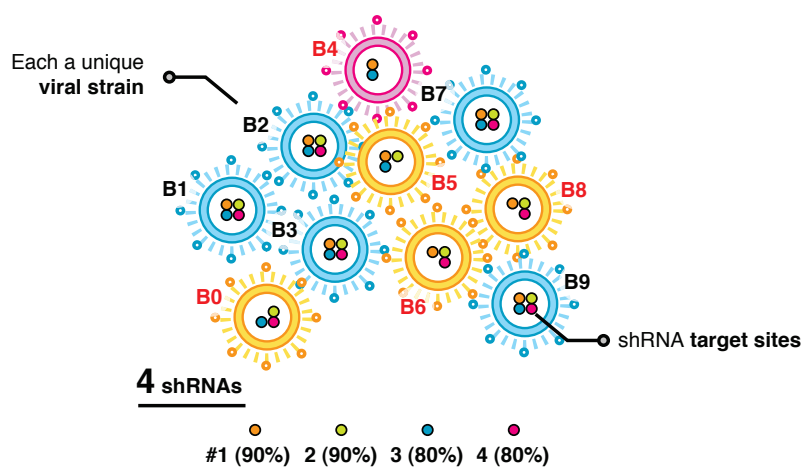

B

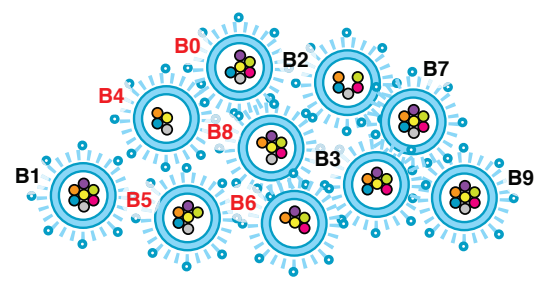

7 shRNAs

$\begin{array}{ccccccc}\circ & \circ & \circ & \circ & \circ & \circ & \circ \\ \text { \#1 }(90 \%) & 2(90 \%) & 3(80 \%) & 4(80 \%) & 5(90 \%) & 6(80 \%) & 7(80 \%)\end{array}$

Figure 1 More than 4 shRNAs are needed to obtain 4 matched to all variants. Models predict that a minimum of 4 shRNAs is needed to prevent the emergence of viral escape mutants, however, this requires 4 shRNAs to be matched to all viral variants spanning the different subtypes. Unless all 4 shRNAs are 100\% conserved across all targeted strains (unlikely) (A), then more than 4 shRNAs will be required to attain at least 4 matched to each different strain (B). By studying the intersecting conservations of combinations as a whole, combinations can be assembled such at at least 4 shRNAs (4+) for a given combination (of > 4 shRNAs) are matched to all relevant strains. 
Table 1 The 10 shRNAs

\begin{tabular}{|c|c|c|c|c|c|c|c|}
\hline$\#^{a}$ & Target & $-2-1^{b}$ & 19 nt. core $+1 / 2$ nt. $^{c}$ & Loop $^{d}$ & $\mathrm{~T}^{\mathrm{e}}$ & $B^{f}$ & ALL \\
\hline 0 & LTR 510-21 & $A A$ & CCCACTGCTTAAGCCTCAATA & ACTCGAGA & & 100 & 70 \\
\hline 1 & LTR 527-21 & $\mathrm{TC}$ & AATAAAGCTTGCCTTGAGTGC & ACTCGAGA & G & 100 & 93 \\
\hline 2 & Gag 533-20 & AG & GAGCCACCCCACAAGATTTA & $\mathbf{T C T C G A G T}$ & & 80 & 70 \\
\hline 3 & Pol 248-20 & AG & GAGCAGATGATACAGTATTA & CCTCGAGC & & 87 & 80 \\
\hline 4 & Pol 2670-21 & GC & AGTACAAATGGCAGTATTCAT & ACTCGAGA & G & 74 & 73 \\
\hline 5 & Pol 2878-20 & $A A$ & GGTGAAGGGGCAGTAGTAAT & TCTCGAGT & & 80 & 74 \\
\hline 6 & Vif 9-21 & $A A$ & CAGATGGCAGGTGATGATTGT & ACTCGAGA & & 92 & 71 \\
\hline 7 & Tat (x1) 140-21 & CT & ATGGCAGGAAGAAGCGGAGAC & ACTCGAGA & A & 77 & 73 \\
\hline 8 & Vpu $143-20$ & AA & GAGCAGAAGACAGTGGCAAT & CCTCGAGC & & 83 & 66 \\
\hline 9 & Env 1428-21 & $\mathrm{AA}$ & TTGGAGAAGTGAATTATATAA & $\mathbf{A C T C G A G A}$ & & 81 & 73 \\
\hline
\end{tabular}

a shRNA reference number (\#) used in this study.

${ }^{\mathrm{b}}$ The two nt. immediately upstream of the $19 \mathrm{bp}$ core target site were taken into consideration when estimating individual shRNA conservations but not included in the shRNA stem, nor used in calculating intersecting conservations.

c The shRNAs had 20 or 21 bp stems built around a 19 bp core placed at the base terminus of the shRNA which was extended by 1 or 2 nt. with target matched sequence at the loop terminus, as indicated in bold (and used when estimating individual shRNA conservations).

${ }^{d}$ If the core was made into a 20 bp hairpin stem, then the last nucleotide of the loop was selected to be the complement of the last nucleotide of the $p+2$ position so that if the processed siRNA product(s) included the the last nucleotide of the loop then it too would be matched to the target (indicated by underline).

e The shRNAs in which the last base of the anti-sense stem was ' $T$ ' also included a 'termination spacer' so as to prevent premature termination via an early run of 'T's. This nucleotide was always the complement of the first nucleotide of the p-1 position (but never a ' $T$ '), so that if included in the processed siRNA product(s) it was also matched to the target.

f $\%$ conservation for the 19 bp core in LANL clade B sequences only.

$9 \%$ conservation for the 19 bp core in ALL LANL sequences, irrespective of clade.

target diversity, with a bias towards selecting highly conserved sequences. The selected shRNAs were either active ( $>50 \%$ activity) or highly active (> 75\% activity), and the average conservation for the central cores (the first 19 nt. of each stem) was 74\% amongst all known sequences and $85 \%$ for clade B subtypes. Our estimates of conservation were as stringent as possible in that we only regarded shRNAs that were fully matched as conserved (i.e. no mismatch tolerance). It may well be that our shRNAs are active against an even greater number of variants than we predict as some shRNAs can retain partial or full activity with some degree of mismatch to their targets. It is interesting to note that the two LTR shRNAs (\#0 and 1) we chose were 100\% conserved in clade B subtypes. It is also interesting that whilst our selection process was entirely independent of prior studies, several of our selected shRNA target sites (e.g. \#3, 4 and 7) are highly similar to those identified by others; see our earlier report for a relevant list $[11,12,37]$.

\section{Transferring shRNAs and confirming target-specificity}

Our 10 shRNAs were first transferred from pSilencer type plasmids [12] as complete expression cassettes (H1 promoter, shRNA region and terminator) into our Lentivirus transfer plasmid setup with an infinitely expandable MCS [38]. This setup enables any number of PCR/ sub-cloned cassettes to be sequentially inserted by using restriction enzymes (REs) that are repeatedly destroyed and simultaneously re-introduced with each round of cloning (Figure 2). The transferred shRNA were each assayed for suppressive activity using 9 different fluorescent reporters matched to each shRNA (n.b. the two overlapping LTR shRNAs, \#0 and \#1, shared the same reporter) (Figure 3a). This was to confirm the specificity of each shRNA to enable us to accurately test the individual activities of simultaneously expressed shRNAs without reporter cross-reactivity. Each shRNA expression plasmid was co-transfected into HEK293a cells with two reporters; the corresponding target-specific GFP fusion and a non-specific AsRed-1 fusion. Target-specific fluorescence was measured 48 hours later, normalized to the fluorescence of the non-specific reporter, and activities were calculated relative to the fluorescence levels of a control plasmid with an empty expression cassette (composed of the $\mathrm{H} 1$ promoter, but no shRNA). All transferred shRNA maintained a comparable level of target-specific activity to that measured previously from the original plasmids, without reporter cross-reactivity (except that expected for \#0 and \#1) (Figure 4).

\section{Selecting combinations to maximize intersecting conservation}

We mathematically assembled the 10 chosen shRNAs into all possible combinations of 4, 5, 6 and 7 different shRNAs with disregard to order. The total number of combinations (k) from a given set size (n) can be found by the combinatorial 'choose function': n!/(k!(n - k)!). For example, 10!/(4!(10-4)!) equalled 210 possible combinations of 4 shRNAs from our selected set of 10 . 


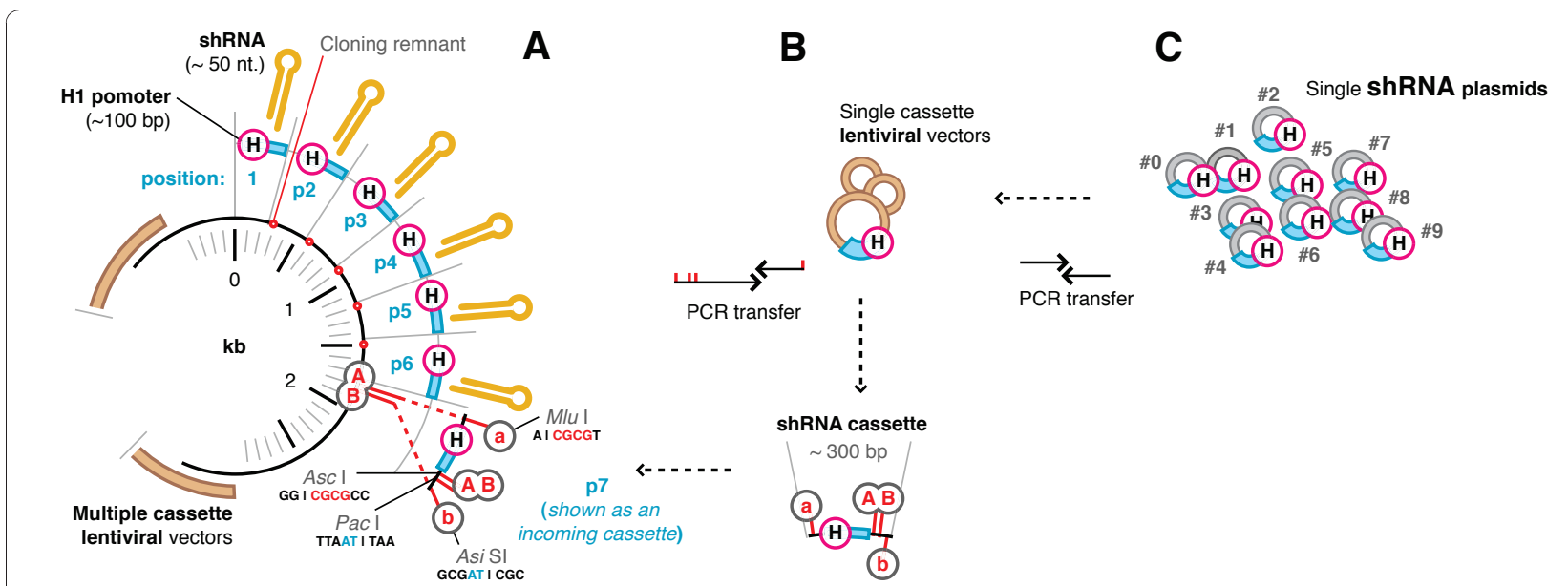

Figure 2 An infinitely expandable cloning strategy. In the example of our multiple cassette cloning strategy shown, a 7th cassette is being inserted into a vector that already has 6 cassettes integrated $(\mathbf{A})$. The incoming donor fragment is a PCR amplified shRNA expression cassette (B) digested with ' $a$ ' (M/u I) and ' $b$ ' (Asi SI) restriction enzymes (REs) which is ligated to the recipient vector opened up with 'A' (Asc I) and 'B' (Pac 1) REs destroying the original ' $a$ ', 'A', $b$ ', and 'B' sites in the process. The newly created vector has the ' $A$ ' and ' $B$ ' sites reconstituted via the incoming donor fragment, ready for insertion of subsequent cassettes. Each shRNA expression cassette included the H1 promoter, shRNA, terminator and some flanking sequence to a total length of $\sim 270$ - 300 bp. (C) All 10 single shRNA expression cassettes were first transferred from pSilencer type plasmids (as assembled in prior work) as complete expression cassettes into single shRNA Lentivirus transfer plasmids (setup with an infinitely expandable MCS as detailed above).

There were 252 possible combinations of 5 shRNAs, 210 of 6 , and 120 of 7 . For each combination we calculated the intersecting conservations, from at least 4 of the component shRNAs, using the first 19 bp of each shRNA stem in accord with our previous target conservation profiling method [12]. Intersecting conservations were calculated using 1224 HIV-1 genome sequences, some with incomplete LTR sequence, obtained from the Los Alamos National Laboratory in 2007 (LANL; http:// www.hiv.lanl.gov). We created 4 sub sets from these: all clade B sequences (229 sequences), all other clades (995), clade B sequences that contained sufficient LTR sequence to analyze combinations including our LTR shRNAs (127), and all other clades with sufficient LTR sequence (549).

There was generally poor intersecting conservations from combinations of 4 shRNAs. Several combinations of 5 had at least 4 shRNAs (4+) conserved in $89-97 \%$ of clade B sequences, though only $40-58 \%$ in all other subtypes (Table 2). But some combinations of 6 and 7 shRNAs had 4+ intersecting conservations of $98-100 \%$ in clade B and 65 - 87\% in all other subtypes. We selected 17 combinations to construct, composed of 6 combinations of $5 \times$ shRNAs, 8 combinations of $6 \times$ shRNAs, and 3 combinations of $7 \times$ shRNAs. Our selection included combinations that excluded LTR shRNAs as there is still some uncertainty surrounding the accessibility of the incoming virus and the LTR as an in-vivo RNAi target [39-41]. Though given the possibility $[37,39,40]$, we also selected combinations that specifically included LTR
shRNAs as they had the highest individual conservation levels. Combinations including overlapping shRNA targets (e.g. shRNAs \#0 and \#1) were discounted. The shRNA order of each combination was chosen to minimize construction steps by creating common subcombinations from the most common shRNAs first. In total, there were 26 combinations assembled including 9 sub-combinations of 4 or less shRNAs required for our 17 final combinations of 5 to 7 shRNAs.

\section{Establishing positional differences in combinations of up to 7 cassettes}

We also created 33 controls, composed of 6 empty cassette combinations of $2-7$, and 27 combinations of a single shRNA (shRNA \#3; Pol 248-20) surrounded by 1 or more empty expression cassettes (in combinations of 2 - 7). In this way our control shRNA could be tested in each position for potential effects by neighboring promoters without competition from other shRNAs for the RNAi machinery. The suppressive activity of the 27 plasmids was tested using the fluorescent reporter assay with the corresponding reporter (Pol-1), across a titrated range of shRNA plasmid amounts from $400 \mathrm{ng}$ to $1 \mathrm{ng}$ (Figure 5). There was a trend towards decreased activity from plasmids with increased cassette number, irrespective of cassette position, which was most apparent for the 6 and 7 cassette plasmids at low doses. Suitable activity was, however, maintained in all variants for the high to mid doses tested (400 - $100 \mathrm{ng}$ ), with respective standard deviations in apparent suppressive activity of 


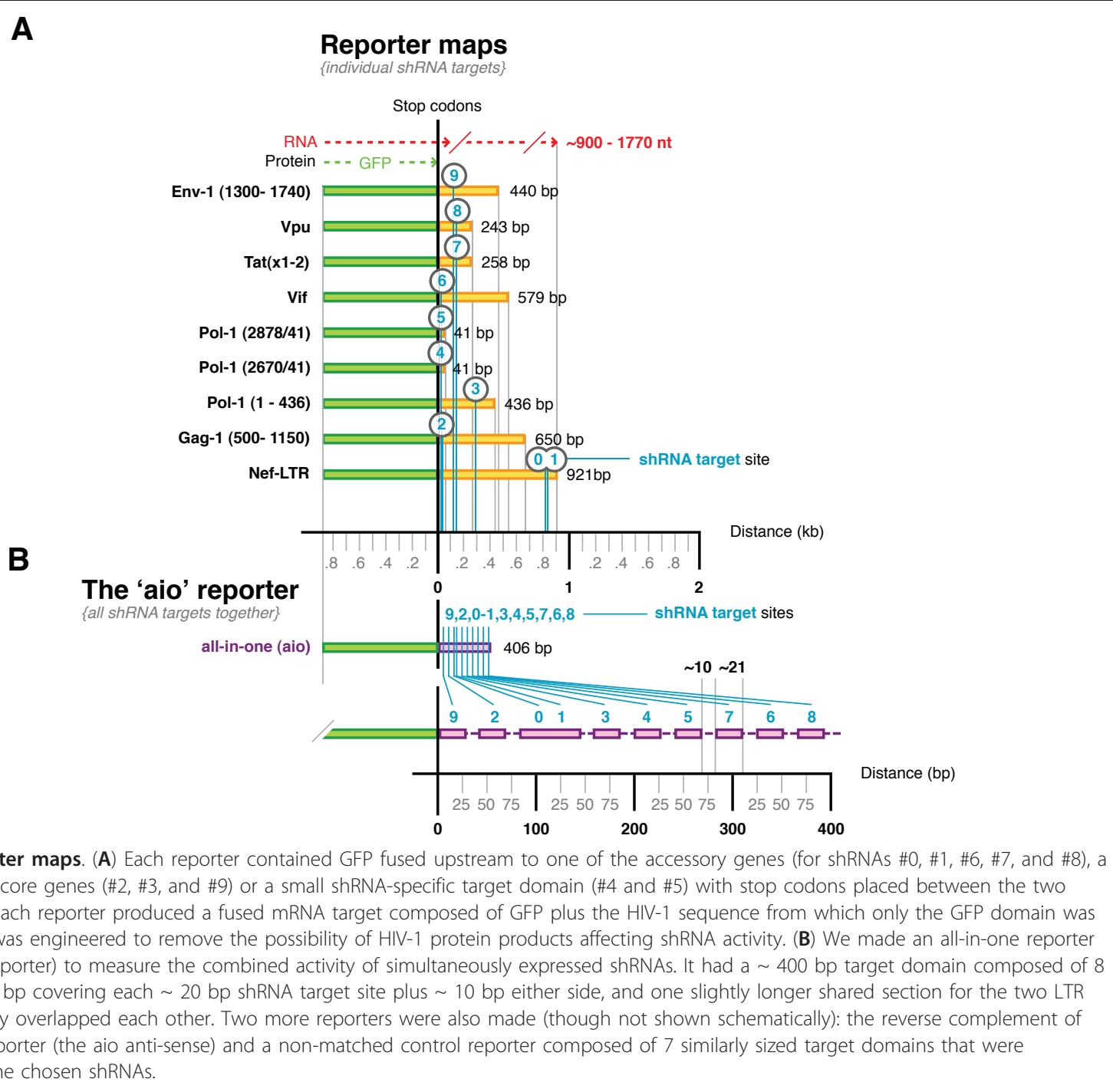

$2 \%$ and $5 \%$ across all positions in all combinations. This confirmed that cassette order had no obvious effect on intrinsic suppressive activity.

\section{Individual activities measured under simultaneous expression}

We measured the individual suppressive activity of each shRNA within all combinations when expressed simultaneously using our fluorescent reporter assay. Every combination and the 10 single shRNA plasmids were separately transfected with each matched reporter (Figure 6). Thus, the apparent suppressive activities likely reflect the individual suppressive contribution of each shRNA to the total. The activities from the single plasmids matched those seen previously. Likewise, the activity of shRNA \#3 (the first position in all combinations) was similar for all combinations and the single
shRNA plasmid. Activities from the second position shRNAs were also comparable to the single shRNA plasmids, however, all shRNA activities from position 3 onwards were notably reduced relative to the single shRNA plasmids. This was most obvious for shRNA $\# 9$ in positions 3,4 and 5 , and \#1 in position 5 ; irrespective of total cassette number. Activities from each shRNA generally clustered, regardless of the position or length of the combination it was present in. For example, while the activities of combinations of 3 to 7 shRNAs measured for shRNA \#7 in positions 3, 5 and 6 (i.e. measured with reporter Tat $\times 12$ ) differed on average more than 2 fold from the \#7 single shRNA, they had a standard deviation of only $4.8 \%$. Our data suggests that shRNA competition may reduce the individual suppressive activities of simultaneously expressed shRNAs, with some sequences more susceptible than others. 


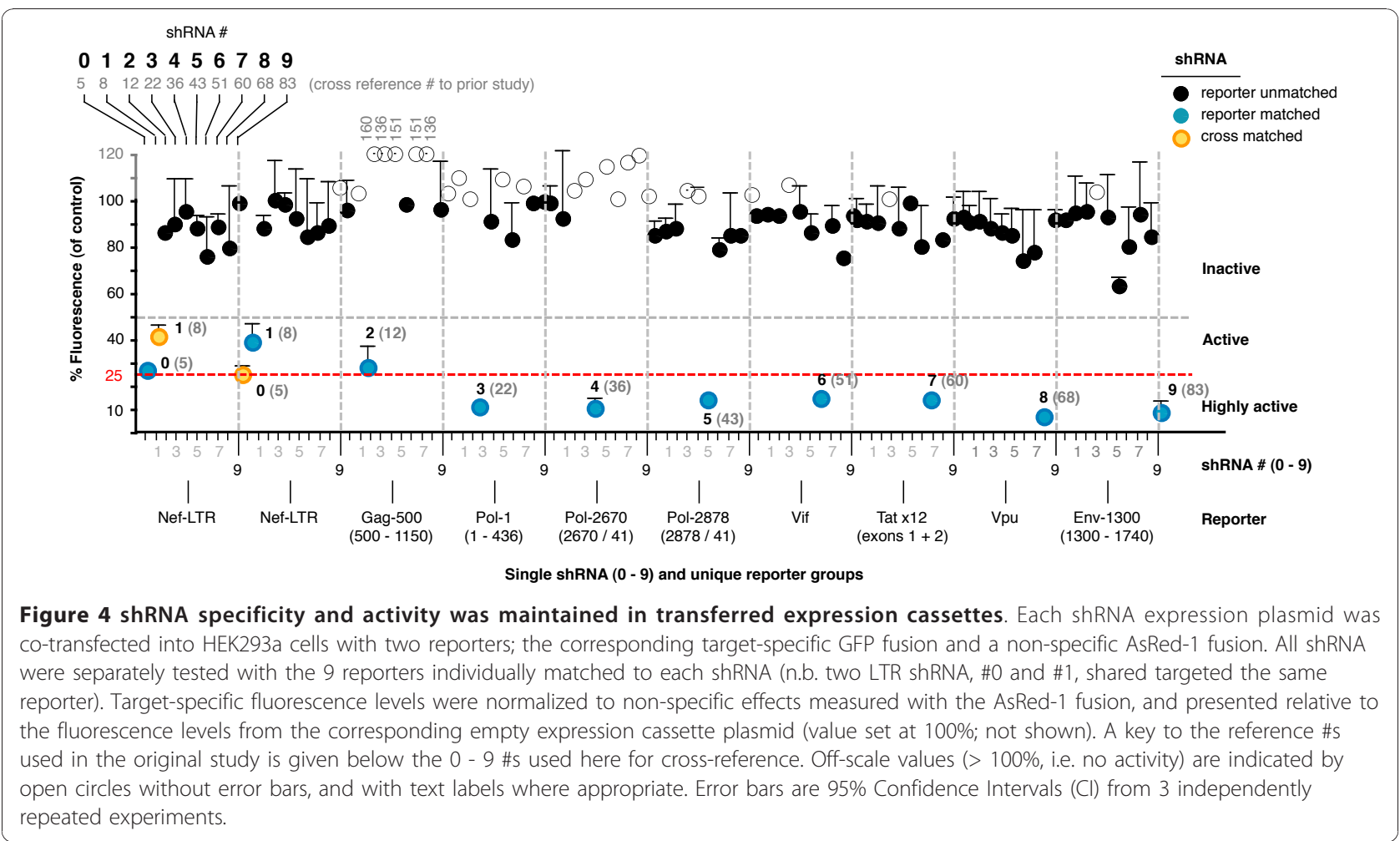

Table 2 Percentage conservations for the combinations

\begin{tabular}{|c|c|c|c|c|c|c|c|c|c|c|c|c|c|c|c|c|c|}
\hline \multirow[b]{3}{*}{$\#^{\mathrm{a}}$} & \multirow[b]{3}{*}{ Combin. ${ }^{c}$} & \multicolumn{4}{|c|}{229 sequences } & \multicolumn{4}{|c|}{995 sequences } & \multicolumn{4}{|c|}{127 sequences } & \multicolumn{4}{|c|}{549 sequences } \\
\hline & & \multicolumn{4}{|c|}{ Clade $B^{d}$} & \multicolumn{4}{|c|}{ Others $^{\mathrm{e}}$} & \multicolumn{4}{|c|}{ Clade B (LTR) ${ }^{\mathrm{f}}$} & \multicolumn{4}{|c|}{ Others $(\mathrm{LTR})^{\mathrm{g}}$} \\
\hline & & $4+$ & $5+$ & $6+$ & $7+$ & $4+$ & $5+$ & $6+$ & $7+$ & $4+$ & $5+$ & $6+$ & $7+$ & $4+$ & $5+$ & $6+$ & $7+$ \\
\hline 5.1 & 3.4.7.2.9 & 89 & 48 & - & - & 54 & 15 & - & - & 91 & 53 & - & - & 58 & 15 & - & - \\
\hline $5.2^{\mathrm{b}}$ & 3.4.7.2.0 & $\mathrm{n} / \mathrm{a}$ & $\mathrm{n} / \mathrm{a}$ & - & - & $\mathrm{n} / \mathrm{a}$ & $\mathrm{n} / \mathrm{a}$ & - & - & 94 & 57 & - & - & 57 & 23 & - & - \\
\hline 5.3 & 3.8.9.2.7 & 92 & 49 & - & - & 40 & 13 & - & - & 94 & 54 & - & - & 41 & 15 & - & - \\
\hline $5.4^{\mathrm{b}}$ & 3.8.5.9.0 & $\mathrm{n} / \mathrm{a}$ & $n / a$ & - & - & $\mathrm{n} / \mathrm{a}$ & $\mathrm{n} / \mathrm{a}$ & - & - & 95 & 64 & - & - & 58 & 20 & - & - \\
\hline 5.5 & 3.8.5.2.9 & 92 & 53 & - & - & 48 & 12 & - & - & 94 & 59 & - & - & 54 & 13 & - & - \\
\hline $5.6^{\mathrm{b}}$ & 3.8.5.2.1 & $\mathrm{n} / \mathrm{a}$ & $\mathrm{n} / \mathrm{a}$ & - & - & $\mathrm{n} / \mathrm{a}$ & $\mathrm{n} / \mathrm{a}$ & - & - & 97 & 65 & - & - & 53 & 10 & - & - \\
\hline $6.2^{\mathrm{b}}$ & 3.4.7.2.0.5 & $\mathrm{n} / \mathrm{a}$ & $\mathrm{n} / \mathrm{a}$ & $\mathrm{n} / \mathrm{a}$ & - & $\mathrm{n} / \mathrm{a}$ & $\mathrm{n} / \mathrm{a}$ & $\mathrm{n} / \mathrm{a}$ & - & 100 & 89 & 51 & - & 81 & 50 & 15 & - \\
\hline 6.3 & 3.8.9.2.7.6 & 100 & 86 & 46 & - & 65 & 26 & 4 & - & 99 & 89 & 49 & - & 67 & 23 & 3 & - \\
\hline $6.4^{b}$ & 3.8.5.9.0.6 & $\mathrm{n} / \mathrm{a}$ & $n / a$ & n/a & - & $\mathrm{n} / \mathrm{a}$ & $\mathrm{n} / \mathrm{a}$ & $\mathrm{n} / \mathrm{a}$ & - & 100 & 88 & 60 & - & 75 & 38 & 5 & - \\
\hline $6.5^{\mathrm{b}}$ & 3.8.5.2.9.0 & $\mathrm{n} / \mathrm{a}$ & $\mathrm{n} / \mathrm{a}$ & $\mathrm{n} / \mathrm{a}$ & - & $\mathrm{n} / \mathrm{a}$ & $\mathrm{n} / \mathrm{a}$ & $\mathrm{n} / \mathrm{a}$ & - & 99 & 94 & 58 & - & 80 & 45 & 13 & - \\
\hline 6.6 & 3.8.5.2.9.7 & 99 & 87 & 43 & - & 68 & 31 & 9 & - & 98 & 92 & 47 & - & 73 & 34 & 10 & - \\
\hline $6.7^{\mathrm{b}}$ & 3.8.5.2.1.7 & $\mathrm{n} / \mathrm{a}$ & $\mathrm{n} / \mathrm{a}$ & $\mathrm{n} / \mathrm{a}$ & - & $\mathrm{n} / \mathrm{a}$ & $\mathrm{n} / \mathrm{a}$ & $\mathrm{n} / \mathrm{a}$ & - & 100 & 94 & 53 & - & 75 & 30 & 7 & - \\
\hline $6.8^{\mathrm{b}}$ & 3.4.7.2.0.6 & $\mathrm{n} / \mathrm{a}$ & $\mathrm{n} / \mathrm{a}$ & n/a & - & $\mathrm{n} / \mathrm{a}$ & $\mathrm{n} / \mathrm{a}$ & $\mathrm{n} / \mathrm{a}$ & - & 99 & 88 & 52 & - & 77 & 40 & 5 & - \\
\hline 6.9 & 3.4 .7 .2 .9 .5 & 98 & 84 & 43 & - & 75 & 45 & 9 & - & 98 & 87 & 47 & - & 79 & 50 & 10 & - \\
\hline $7.3^{\mathrm{b}}$ & $3.8 \cdot 9 \cdot 2 \cdot 7 \cdot 6.0$ & $\mathrm{n} / \mathrm{a}$ & $n / a$ & n/a & n/a & $\mathrm{n} / \mathrm{a}$ & $\mathrm{n} / \mathrm{a}$ & $\mathrm{n} / \mathrm{a}$ & $\mathrm{n} / \mathrm{a}$ & 100 & 98 & 89 & 48 & 87 & 57 & 22 & 3 \\
\hline 7.5 & $3.8 \cdot 5 \cdot 2 \cdot 9.7 .6$ & 100 & 99 & 81 & 41 & 84 & 56 & 18 & 3 & 100 & 98 & 84 & 44 & 85 & 58 & 17 & 2 \\
\hline 7.7 & 3.4 .7 .2 .9 .5 .6 & 100 & 96 & 80 & 40 & 86 & 64 & 31 & 3 & 100 & 94 & 83 & 43 & 86 & 64 & 34 & 2 \\
\hline
\end{tabular}

${ }^{a}$ Combination reference number (\#) used in this study.

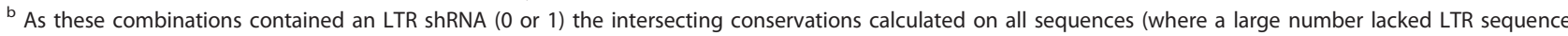
coverage), were not applicable ( $\mathrm{n} / \mathrm{a})$; only the LTR containing subset were used, as shown.

' The component shRNAs (in order of arrangement) for each combination.

${ }^{d}$ The clade B sequences only.

${ }^{\mathrm{e}}$ The sequences of all other clades (excluding clade B).

${ }^{f}$ The subset of clade B sequences that contain LTR sequence coverage.

${ }^{g}$ The subset of all other sequences (non B) that contain LTR sequence coverage. 


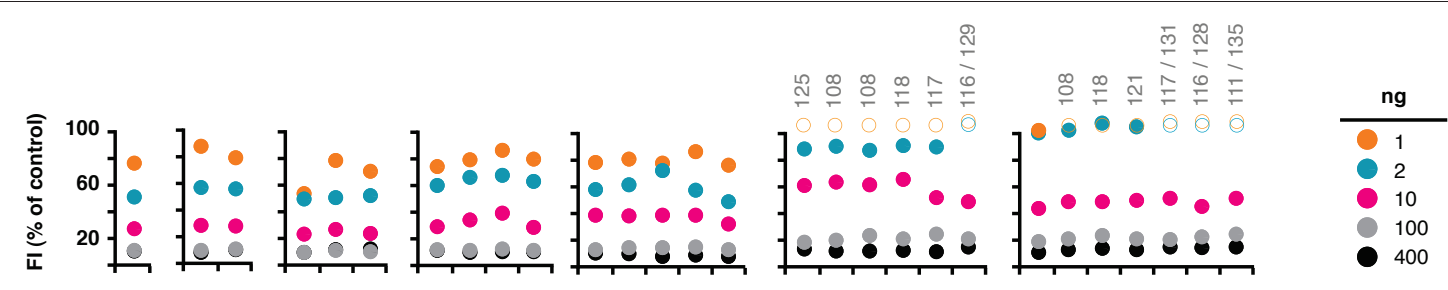

shRNA \#3 in all positions (of all lengths) surrounded by empty expression cassettes (positions reflect the placement of shRNA \#3 )

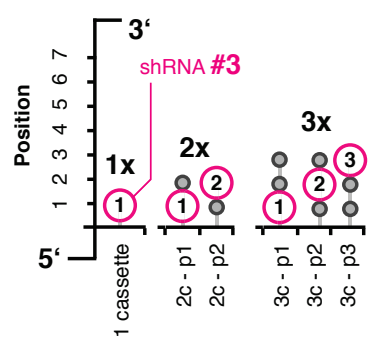

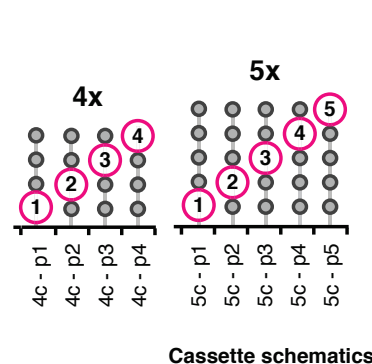

Cassette schematics

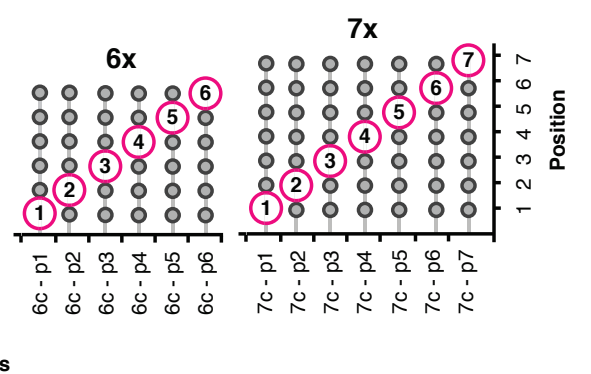

\#)

Cassette position being measured (the sole shRNA cassette; shRNA \#3)

- Empty cassette position

Figure 5 Cassette number and position effects on suppressive activity. Twenty seven control plasmids, each containing a single shRNA expression cassette (shRNA \#3) plus 1 or more empty expression cassettes for all possible 2, 3, 4, 5, 6 and 7 cassette plasmids were tested with the fluorescent reporter assay using the matched Pol-1 (1 - 436) reporter across a titrated range of shRNA plasmid amounts from $400 \mathrm{ng}$ to $1 \mathrm{ng}$. The activity of each combination was calculated as a\% of the fluorescence from the corresponding control of same cassette number and $\sim$ length but composed entirely of empty expression cassettes (values set at 100\%; not shown). Off-scale values are indicated by open circles.

\section{Building all-in-one reporters}

We made a new all-in-one reporter (the aio sense reporter) to measure the combined or total activity of all shRNAs within each combination acting in concert against a single target transcript. This reporter had a $400 \mathrm{bp}$ target domain composed of fused target sections for our 10 chosen shRNAs (Figure 3b). There were 8 sections of $\sim 40 \mathrm{bp}$ covering each $\sim 20 \mathrm{bp}$ target site plus $\sim 10$ bp either side, and one slightly longer shared section for the two overlapping LTR targets. Two additional reporters were also made. One was the reverse complement of the aio sense reporter (the aio antisense) designed to measure suppressive activity of the siRNA passenger strand derived from the anti-sense shRNA stem. The other was a non-matched control reporter composed of 7 similarly sized target domains that were unmatched to the chosen shRNAs. To go with this last reporter, we assembled a series of 7 corresponding non-matched single shRNA controls and used them to make 2, 3, 4, 5, 6 and 7 cassette control combinations. The sequences of the single shRNA controls were derived from the backwards sequence of shRNAs $\# 3$, \#8, \#9, \#2, \#7, \#6 and \#0. In this way they were unmatched to the aio reporters yet had identical nucleotide compositions (but in reverse order) to retain similar thermodynamic profiles.

\section{Combined activities measured with an all-in-one reporter}

All single shRNA, combinations, and non-matched control plasmids were separately transfected with all three reporters (Figure 7a) (Additional file 1 for the control data). The aio sense reporter activities for the 10 single shRNA differed slightly in magnitude to that seen with the previous reporters, but still followed the same relative pattern. shRNAs \#0, \#1 and \#2 were the least active. Interestingly, the aio anti-sense reporter showed that shRNAs \#1, \#7 (especially) and \#8 were being at least partly processed so that the passenger strand was being loaded into RISC. The activity of shRNA \#1 was particularly poor, with the passenger strand exhibiting greater suppressive activity than the guide strand. The activities for all combinations of 2 through to 7 cassettes were similar to each other and the activities of the most active single shRNA (measured with the aio sense reporter). It may be that this was close to the highest silencing level achievable with the aio reporter under the current conditions of our assay system. There was no notable passenger strand activity from any combination. In all cases the single shRNAs and combinations exhibited no notable non-specific effects on the control reporter. The backwards control shRNAs showed the expected reverse trend, with no effect on either aio reporter, but some suppression of the corresponding 


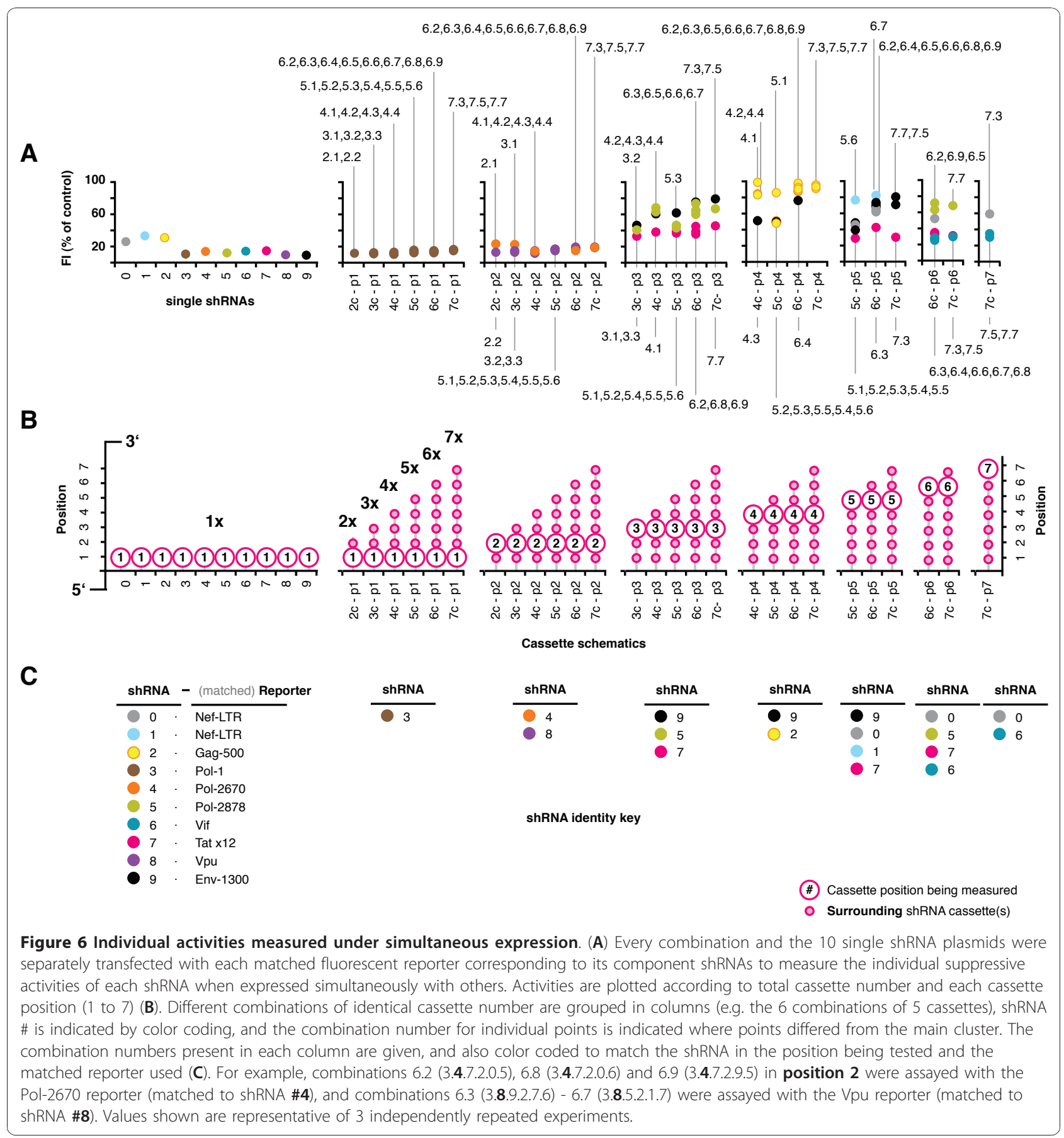

control reporter. Activity levels were spread across the inactive to active classification groups as the backwards control shRNAs were neither designed nor previously selected for activity. Interestingly, the combined suppressive activities of these mediocre shRNAs at maximum dosage was not additive, i.e. resulting in greater total suppressive activity. Again, no control combination exceeded the activity of the most active single shRNA.
Titrations to look at sub-saturating differences between combinations

We repeated the transfections of the aio sense reporter with each combination, but titrated the amount of single shRNA or combination plasmid from $400 \mathrm{ng}$ to $1 \mathrm{ng}$ to determine if there was a sub-saturating point at which larger combinations were more active than smaller ones (Figure 7b). The suppressive activities at the higher 


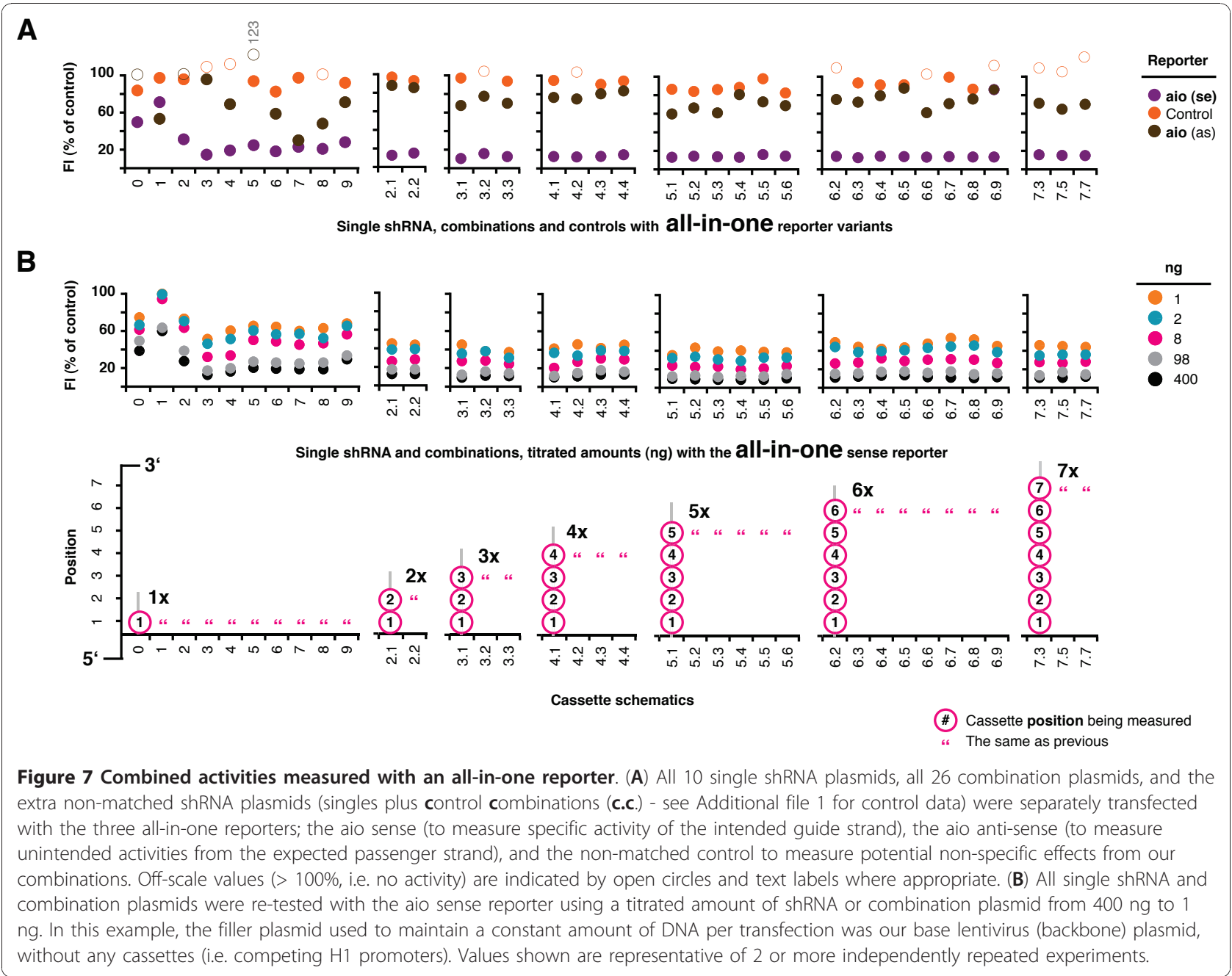

dosages were similar to that seen previously, and all combinations were more active than any of the single shRNAs at all titration points. This showed that one or more shRNAs in combinations can exhibit an increased combined suppressive effect at sub-saturating expression levels compared to any one of the component single shRNAs. However, there were no obvious differences between combinations of different number, with an average standard deviation of only $2 \%$ at all titration points.

We also measured the titrated activities using shRNAspecific reporters and a series of related combinations (2.2, 4.2 and 6.3) and their corresponding single shRNA plasmids (\#3, \#8, \#9, \#2, \#7, and \#6) (Figure 8). The activities of the selected single shRNAs at maximum dosage was generally higher with the shRNA-specific reporters (cf. the aio reporter), excepting \#2, and clustered closely for both reporter types. The individual suppressive activities of each shRNA expressed simultaneously with all others was reduced relative to activities from the corresponding single shRNA plasmids at all titration points, with the exception of shRNA \#3 (position 1). shRNAs \#9 and \#2 in the central positions (3 and 4) displayed notably impaired contributing activities when expressed in combination. The positional overlay connecting the maximum dosages showed the same pattern as seen previously when testing all combinations. Likewise, the combined activities for all combinations were similar at all titration points, but generally greater than the component single shRNAs at lower dosages.

\section{Discussion}

In this study we aimed to mathematically assemble, select and test combinations of highly-conserved antiHIV shRNAs to find those with the highest intersecting conservations of $4+$ shRNAs across all known viral strains. Importantly, we have shown that it is possible, with careful consideration of the individual and intersecting conservations of combinations of shRNAs, to 


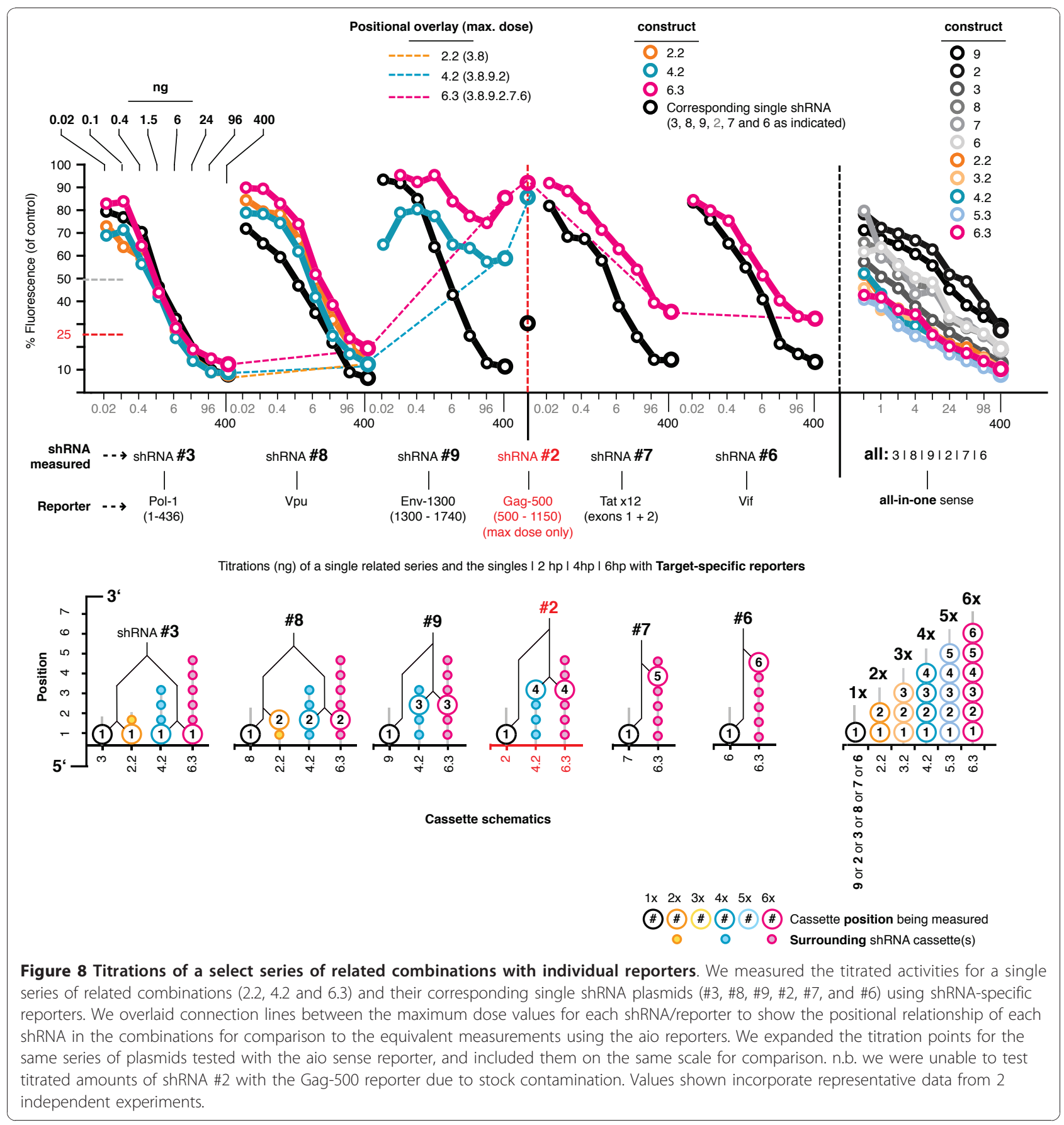

assemble shRNA combinations against entire subtypes. Even when selecting the most conserved individual shRNAs we were unable to identify a combination of 4 that was fully matched to all variants analyzed, as there were not 4 non-overlapping shRNAs that were $100 \%$ conserved. But with selected combinations of 7 shRNAs we could attain at least 4 shRNAs matched to $100 \%$ of clade B subtypes, and up to $87 \%$ of all other clades - a highly significant finding. In demonstration of the need to consider intersecting conservations, 5 of our highest individually conserved shRNAs (\#0|1,3, 6, 8, and 9) had an intersecting conservation for clade $B$ subtypes that was $6 \%$ lower than other possible combinations (91 vs. 97\%). Also, we found that different combinations were better suited to different subtypes. For example, the combinations of 5 shRNAs with the highest intersecting conservations for clade B subtypes (96-97\%) had conservations for all other subtypes that were between at 
least 16 and $28 \%$ less (34 - 53\%) than others most highly conserved for all other subtypes $(62-69 \%)$. Thus, subtype-specific intersecting conservations should also be considered if tailoring combinations to localities with a subtype bias.

The data from our series of combinations containing a single copy shRNA \#3 surrounded by empty expression cassettes revealed that although there was some reduction in activity for combinations of 6 and 7 shRNAs, there was no obvious difference in the activities of the different cassette positions for any combination length. When we assembled combinations of multiple shRNAs and tested the individual activities of the component shRNAs, we found that the shRNAs in positions 1 and 2 were the most active (\#3,4 and 8), and similar in activity to the corresponding single shRNAs for all combinations. However, shRNAs in positions 3 to 7 exhibited a marked reduction in activity compared to their single shRNA counterparts, irrespective of combination length or composition, and notably to similar levels for each shRNA. Some of the corresponding single shRNAs were highly active (\#5, 6, 7 and 9), and just as active as the single shRNAs corresponding to positions 1 and 2 . We conclude that the shRNAs located in positions 3 to 7 were likely more susceptible to a reduction in suppressive activity due to competition than the shRNAs in positions 1 and 2 . If so, the effect of competition on individual suppressive activities may, at least in part, be sequence dependent. Future screens for component shRNAs for assembly in combinations may benefit from the inclusion of an additional test to measure individual suppressive activity when expressed in combination. However, it is also possible that there may be some additional positional influence contributing to our observations from the shRNA combinations that was not apparent from our shRNA \#3 control series. This possibility could be examined with further work including the assembly of permutations of the combinations to place each shRNA in several different positions whilst being surrounded by other shRNAs.

Potential competition for the available RNAi machinery between simultaneously expressed shRNAs (and host cell miRNAs) also needs to be considered. It is likely that expression level will need to be carefully controlled to maximize the specific activities of the antiHIV shRNAs, yet minimize the impact on the natural miRNA processes. Another potential issue is that the shRNAs not matched to the given strain may reduce the individual impacts of those that are matched [16,29], which implies that combination size should be kept to a minimum. We also noted from our positional testing experiments that combinations of $>5$ shRNAs began to show reduced activity from all cassette positions due to increased size alone. It may be a more effective gene therapy strategy to use several smaller combinations of $\sim 5$ shRNAs selected with intersecting conservations specific to the strains that predominate geographical locations, rather than a single larger combination to cover all variants simultaneously.

Although multiple repeated expression cassettes is currently a most useful co-expression strategy for multiple shRNAs, it has some shortcomings, including concerns regarding long-term stability and efficacy [28]. We chose this system here not because we necessarily think that it will be the best in the long run, but because at the time of design it was the most advanced method compatible with out goals. We and others have found that large increases in vector size may affect vector production $[23,33,42]$. It is possible that the impact of these issues could be reduced or eliminated with fewer cassettes, further supporting the use of the smallest effective combination size. Current designs like ours that use unregulated pol III promoters may also have to be redesigned with regulated or inducible expression units for in vivo applications (e.g. [22]). Our choice of the H1 promoter (compared to say, the U6 promoter) was because of our extensive experience with this promoter and its common use for expressing shRNAs. Furthermore, there are reports that U6 expressed shRNAs may cause an adverse response in vivo, though this was overcome by using different pol II promoter types [43]. Other co-expression methods may be ultimately better suited for our gene therapy, such as single transcripts composed of multiple target domains. Several such designs have been tested by others, though in some cases it has been difficult to attain activity from more than 3 domains $[34,44]$. Also, their use will also likely be complicated by the probability that each domain is processed in a different manner to the component shRNAs. As with all co-expression methods, one should remain mindful of the possibility of unexpected offtarget effects. Additionally, all uses of a lentivirus delivery system for a HIV sequence-specific treatment such as shRNA should, at some stage, consider the possibility that the shRNA used may interfere with the production of the lentivirus itself. We, however have not yet found this problematic [45] and the extent to which this would be a practical problem on a commercial-scale is as yet undetermined, though there are several ways in which it may be overcome.

We have since begun testing our combinations in HIV replication assays and discovered that repeat-mediated deletion can result in the loss of one or more complete expression cassettes [45]. We have extensively characterised the frequency of this phenomenon, and modeled its likely impact on treatment success. Surprisingly, we have found that even significant amounts of deletion are unlikely to affect the treatment outcome. Thus, the next 
step is to test the combinations for their capacity to suppress the emergence of escape mutants over time, similar to other studies $[11,16]$. This is the critical assessment of activity to confirm the theoretical advantages of multiple shRNAs frequently speculated upon [46-48], and supported by mathematical modeling [49]. In addition to this, the long-term stability of our combinations, and potential cellular affects will need to be assessed. Direct measurements of expression level could also be beneficial for refinement of our configurations and better understanding potential differences resulting from cassette positions (and how these operate over time under stable expression). Finally, reformulated combinations could be investigated using a wider range of shRNAs reanalyzed to include the following: (1) very highly active, e.g. > 95\%, (2) tested suppressive activity when expressed in combination, and (3) high strand bias to reduce competition with the active strand. All these factors will be important to maximize the impact from combinations of the smallest effective size.

In summary, we have assembled the most clinically relevant shRNA combinations to date, and characterized the intrinsic suppressive activities of both the component shRNAs and the combinations as a whole. While we found all combinations had high combined suppressive activities, there were also large changes in the individual activities of the component shRNAs. Though a useful start, it is not the physical configuration of our multiple cassette method that is the key finding. Rather, we have demonstrated that it is possible to assemble combinations of $4+$ highly active, highly conserved shRNAs that can cover all known variants for entire subtypes (e.g. clade B). Such combinations could thus be tailored to geographical sub-type prevalence with the theoretical potential to prevent the emergence of therapy-resistant strains. By extension, our work suggests that it may also be possible to assemble just a few combinations for complete global coverage of all known HIV-1 variants; something as yet unachievable by any other means.

\section{Methods}

\section{Single target and all-in-one (aio) fluorescent reporter constructions}

The HIV-1 fluorescent protein-target fusion reporter plasmids were constructed using EGFP (from pd4-d4EGFP-N1, BD Biosciences), AsRed1 (from pAsRed1-C1, BD Biosciences) and HIV-1 sequences PCR amplified or synthetically generated from variant NL4-3 [Genbank:AF324493] (Additional file 1). Each single target reporter contained GFP fused upstream to one of the accessory genes (for shRNAs $\# 0, \# 1, \# 6$, \#7, and \#8), a fragment of the core genes (\#2, \#3, and \#9) or a small shRNA-specific target domain (\#4 and \#5) with stop codons placed between the two domains so that a fused mRNA target was transcribed but only the GFP domain was translated. The aio reporters were created using custom generated target domains (GenScript; http://www.genscript.com) composed of 9 target domains matched to our 10 shRNAs, or 7 unmatched control domains (for the control reporter) which were transferred via PCR into our EGFP reporter base plasmids. The elements of the $406 \mathrm{bp}$ combined target domain synthesized for the aio sense reporter were (Xho I - Bgl II ffor cloning\} - \#9 - \#2 - \#0|1 - \#3 - \#4 \#5 - \#7 - \#6 - \#8 - Sal I - Bam HI \{for cloning\}).

\section{Assembly of multiple shRNA expression plasmids}

The infinitely expandable multiple cloning site was assembled by annealing two complementary synthetic oligonucleotides, and inserting it into a recipient plasmid (a $7 \mathrm{~kb}$ carrier plasmid encoding a lentiviral transfer vector) which was a derivative of $\mathrm{pKC}\left(\mathrm{ro}^{-}\right) \mathrm{MND} . \mathrm{MCS}$ obtained from Cell Genesys, as previously described [38]. The 10 chosen shRNA targets, and the original individual shRNA expression plasmids used here as PCR templates were selected and constructed as previously described [12,50] (Additional file 1). The multiple shRNA combinations were assembled in our lentivirus plasmids by sequentially inserting each PCR amplified shRNA expression cassette. Because many shRNAs were present in several combinations, we were able to minimize construction effort by creating shared subcombinations from the most common shRNAs first. As such, all combinations began with shRNA \#3, and had either $\# 4$ or $\# 8$ in position $2, \# 5$, \#7 or \#9 in position 3, and so on, becoming increasingly diverse for each combination. The PCR primers used to amplify the shRNA expression cassettes were common to all plasmids; the forward primer (5'-3'): GC \{seat $\}$ ACGCGT $\{M l u I\}$ GTT TTC CCA GTC ACG AC \{binding site $\}$, and the reverse primer (5'-3'): GC \{seat $\}$ GCGATCGC $\{$ Asi SI\} TTAATTAA $\{$ Pac I $\}$ CCCGGG spacer $\}$ GGCGCGCC \{Asc I\} GCT GCA ATA AA CAA GTT A $\{$ binding site\}. Each PCR consisted of the core 2 primers $(20$ pmol each), $1 \times$ PCR II buffer (Roche) $2.5 \mathrm{mM} \mathrm{MgCl}_{2}, 10 \mathrm{mM}$ dNTPs (each), $100 \mathrm{ng}$ of template, $0.5 \mu \mathrm{l}$ AmpliTaqGold (Roche), and $\mathrm{H}_{2} \mathrm{O}$ to a final volume of $50 \mu \mathrm{l}$. Each PCR was cycled at $1 \times$ : $94^{\circ} \mathrm{C}$ for $10 \mathrm{~min}$., $35 \times$ : $94^{\circ} \mathrm{C}$ for $30 \mathrm{sec}$. $55^{\circ} \mathrm{C}$ for $30 \mathrm{sec}$. $72^{\circ} \mathrm{C}$ for $30 \mathrm{sec}$, and $1 \times 72^{\circ}$ $\mathrm{C}$ for $10 \mathrm{~min}$. End digestions were conducted directly in the PCR mix (after cycling) by adding $5 \mu \mathrm{l}$ of $10 \times$ BSA, $1 \mu \mathrm{l}$ each of $M l u \mathrm{I}$ and Asi SI and incubating @ $37^{\circ} \mathrm{C}$ for a minimum of $1 \mathrm{hr}$. All restriction enzymes were sourced from New England Biolabs (NEB). Digested cassettes were separated on $2 \%$ TAE agarose gels, gel extracted (Qiagen Gel Extraction kit) and eluted in $35 \mu \mathrm{l}$ of $\mathrm{H}_{2} \mathrm{O}$. Recipient plasmids were prepared by 
digestion of $\sim 10 \mu \mathrm{g}$ with $1 \mu \mathrm{l}$ each of Asc I and Pac I, NEB 4 buffer, BSA plus $\mathrm{H}_{2} \mathrm{O}$ to a final volume of $50 \mu \mathrm{l}$ and incubation at $37^{\circ} \mathrm{C}$ overnight. This was followed by heat inactivation $\left(65^{\circ} \mathrm{C}\right.$ for $20 \mathrm{~min}$.) and de-phosphorylation by adding $5 \mu \mathrm{l}$ Antarctic Phosphatase (NEB), $5 \mu \mathrm{l}$ buffer and incubating at $37^{\circ} \mathrm{C}$ for a minimum of $1 \mathrm{hr}$. Antarctic Phosphatase was heat inactivated $\left(65^{\circ} \mathrm{C}\right.$ for 10 min) prior to separation on $1 \%$ TAE agarose gels and gel extraction (performed as already described). Single donor cassettes were ligated into the linearized recipient plasmid using $4 \mu \mathrm{l}$ of vector, $6 \mu$ l of shRNA cassette, 10 $\mu \mathrm{l}$ of Quick DNA ligase buffer and $1 \mu \mathrm{l}$ of Quick DNA ligase (NEB). The ligations were incubated at room temperature for $5 \mathrm{~min}$., and then purified using the QIAgen PCR Purification kit by mixing with 5 volumes $(105 \mu \mathrm{l})$ of Buffer $\mathrm{PB}$, and eluting in $35 \mu \mathrm{l} \mathrm{H}_{2} \mathrm{O}$. Ligated products were transformed by electroporation under standard conditions, and positive colonies were identified by a direct colony PCR technique using Pfu polymerase. All plasmids were propagated in GT116 E. coli cells; a cell line specifically developed for the replication of hairpin containing plasmids (Invivogen). DNA was extracted (Hi-speed Maxi-prep Kit, Qiagen), quantitated in triplicate (Nanodrop) and sequence confirmed via automated sequencing.

\section{Pfu-based PCR screening and gel electrophoresis}

As previously described [38], inserted donor fragments were screened by gel analysis of PCR amplicons made using primers that flanked the MCS; forward (5'-3'): AGT TCT GCA CTC GGC CTC TG, and reverse (5'3'): CCA TGG TCT GCA GTC GCT AG. These were positioned $38 \mathrm{bp}$ upstream and $21 \mathrm{bp}$ downstream (inclusive). The optimized Pfu-based PCR screening method consisted of the primers (20 pmol each), $1 \times$ Pfu Ultra II HS buffer (Stratagene), $3.5 \mathrm{mM} \mathrm{MgCl}_{2}$ (total), $10 \mathrm{mM}$ dNTPs (each), $10 \mathrm{ng}$ of template, 2.5 $\mu \mathrm{l}$ DMSO (5\%), $0.5 \mu \mathrm{l} \mathrm{Pfu}$ Ultra II HS (Stratagene), and $\mathrm{H}_{2} \mathrm{O}$ to a final volume of $50 \mu \mathrm{l}$. Each PCR was cycled at $1 \times$ : $95^{\circ} \mathrm{C}$ for $2 \mathrm{~min}$., $35 \times$ : $95^{\circ} \mathrm{C}$ for $20 \mathrm{sec} .66^{\circ} \mathrm{C}$ for 20 sec. $\mid 72^{\circ} \mathrm{C}$ for $0.5-4 \mathrm{~min}$. (depending upon template length), and $1 \times 72^{\circ} \mathrm{C}$ for $3 \mathrm{~min}$. Samples were electrophoresed on 1\% TAE agarose gels plus $0.01 \%$ SYBRSafe stain (Invitrogen) at $200 \mathrm{~V}$ (limiting) for $\sim 60 \mathrm{~min}$. using a $150 \times 245 \mathrm{~mm}$ tray, $3 \mathrm{~mm}$ wells with a Bio-Rad sub-cell model 192 apparatus.

\section{Sequence confirmation}

The sequence of each sub- and all final combinations were verified by automated sequencing, which was technically challenging due to high amounts of secondary structure resulting in poor reads, and the lack of unique binding sites due to repeated sequence shared amongst all cassettes. It required the use of the external primers previously described for the PCR screening method (binding to flanking plasmid regions) and shRNA specific primers (designed to overlap unique stem regions) to create contiguous partial sequence reads that could be assembled into single continuous reads using CodonCode Aligner for Mac OS $\times$ (CodonCode Corporation; http://www.codoncode.com).

\section{Fluorescent reporter assay}

Each shRNA expression plasmid was co-transfected with two reporters; the corresponding target-specific GFP fusion and a non-specific AsRed-1 fusion. The non-specific reporter was typically an AsRed-1-Vpr fusion, since we selected no Vpr shRNA targets. Low passage no. HEK293a cells (sourced from the American Type Culture Collection) [ATCC:CRL-1573] were seeded at a density of $5 \times 10^{5}$ cells per well ( 6 well plates; $2 \mathrm{ml}$ of medium). Cells were transfected 1 day later using $1 \mu \mathrm{g}$ of total DNA (400 $\mathrm{ng}$ of shRNA expression plasmid, $300 \mathrm{ng}$ of target plasmid and 300 ng of control plasmid) with $4 \mu \mathrm{l}$ of Lipofectamine 2000 (Invitrogen) in OptiMEM (Invitrogen) to a total volume of $100 \mu \mathrm{l} /$ well. For titration experiments up to $400 \mathrm{ng}$ of shRNA expression plasmid was supplemented with the appropriate amount of the corresponding empty expression control plasmid to a total amount of $400 \mathrm{ng}$ as indicated. Cells were analyzed by flow cytometry 48 hours later (FACsCalibur and CellQuest Pro for Mac OS X, BD Biosciences). Target-specific suppression was measured as a decrease in green fluorescence (FL1 channel) and non-specific effects were measured as a change in red fluorescence (FL2 channel). The Fluorescence Index (FI) of cells in each channel was calculated by multiplying the geo mean of fluorescence by the percentage of cells that were fluorescent (only those cells gated above background). The FI of FL1 (green, target-specific activity) was normalized to remove non-specific effects (FI of FL1 normalized to the FI of FL2) and was expressed as a percentage of the FI of cells transfected only with the control plasmid that expressed no shRNA. Each experiment included a mock transfection (i.e. no DNA) and an off-target shRNA control (to verify that on-target shRNA suppression was sequence-specific), both of which behaved as expected and both of which were omitted from the graphs for clarity.

\section{Additional material}

Additional file 1: Contains additional data for the control sequences, detailed conservation profiles for the 10 selected shRNAs, detailed construction methods for the single shRNA expression plasmids and sequences for the reporter (mapped with the chosen shRNAs) and control sequences. 


\section{Acknowledgements}

Thanks to Associate Prof. John Murray at UNSW for the modeling data, Dr. Michael Poidinger at JJR for help in mathematically assembling the combinations, Dr. Toby Passioura at JJR for helpful discussions, and Cell Genesys for providing the lentivirus expression plasmids. This work was funded by JJR. Figures were prepared by http://www.madebyglen.com

\section{Authors' contributions}

GJM and TLA conceived the experiments. GJM, JLG, YY and AT constructed the plasmids and performed the fluorescent reporter assays. GJM and TLA analyzed and interpreted the results. GJM. wrote the manuscript. All authors have read and approved the final manuscript.

\section{Competing interests}

This work was done by employees of Johnson and Johnson Research (JJR), for JJR.

Received: 19 August 2010 Accepted: 13 January 2011

Published: 13 January 2011

\section{References}

1. Kuiken C, Korber B, Shafer RW: HIV sequence databases. AIDS Rev 2003, 5:52-61.

2. LANL: Los Alamos National Laboratory HIV sequence database. 2006.

3. Yeni P: Update on HAART in HIV. J Hepatol 2006, 44(Suppl 1:):S100-103.

4. Zamore PD, Haley B: Ribo-gnome: the big world of small RNAs. Science 2005, 309:1519-1524.

5. Hannon GJ, Rossi JJ: Unlocking the potential of the human genome with RNA interference. Nature 2004, 431:371-378.

6. Stevenson M: Therapeutic potential of RNA interference. N Engl J Med 2004, 351:1772-1777.

7. Dickins RA, Hemann MT, Zilfou JT, Simpson DR, Ibarra I, Hannon GJ, Lowe SW: Probing tumor phenotypes using stable and regulated synthetic microRNA precursors. Nat Genet 2005, 37:1163-1165.

8. Rubinson D, Dillon C, Kwiatkowski A, Sievers C, Yang L, Kopinja J, Rooney D, Ihrig M, McManus M, Gertler F, et al: A lentivirus-based system to functionally silence genes in primary mammalian cells, stem cells and transgenic mice by RNA interference. Nat Genet 2003, 33:401-406.

9. Lee M, Coburn G, McClure M, Cullen B: Inhibition of human immunodeficiency virus type 1 replication in primary macrophages by using Tat- or CCR5-specific small interfering RNAs expressed from a lentivirus vector. J Virol 2003, 77:11964-11972.

10. Naito, Nohtomi, Onogi, Uenishi, Ui-Tei, Saigo, Takebe: Optimal design and validation of antiviral siRNA for targeting HIV-1. Retrovirology 2007, 4:80.

11. ter Brake O, Konstantinova P, Ceylan M, Berkhout B: Silencing of HIV-1 with RNA interference: a multiple shRNA approach. Mol Ther 2006, 14:883-892.

12. Mcintyre G, Groneman J, Yu Y, Jaramillo A, Shen S, Applegate T: 96 shRNAs designed for maximal coverage of HIV-1 variants. Retrovirology 2009, 6:55.

13. Das AT, Brummelkamp TR, Westerhout, Vink M, Madiredjo M, Bernards R, Berkhout B: Human immunodeficiency virus type 1 escapes from RNA interference-mediated inhibition. Journal of Virology 2004, 78:2601-2605.

14. Boden, Pusch, Lee, Tucker, Ramratnam : Human immunodeficiency virus type 1 escape from RNA interference. Journal of Virology 2003, 77:11531-11535.

15. Westerhout EM, Ooms M, Vink M, Das AT, Berkhout B: HIV-1 can escape from RNA interference by evolving an alternative structure in its RNA genome. Nucleic Acids Res 2005, 33:796-804.

16. Nishitsuji : Effective Suppression of Human Immunodeficiency Virus Type 1 through a Combination of Short- or Long-Hairpin RNAs Targeting Essential Sequences for Retroviral Integration. Journal of Virology 2006 80:7658-7666.

17. Ter brake O, Berkhout B: A novel approach for inhibition of HIV-1 by RNA interference: counteracting viral escape with a second generation of siRNAs. Journal of RNAi and Gene Silencing 2005, 1:56-65.

18. Wilson J, Richardson C: Hepatitis C virus replicons escape RNA interference induced by a short interfering RNA directed against the NS5b coding region. J Virol 2005, 79:7050-7058.

19. Leonard JN, Schaffer DV: Computational design of antiviral RNA interference strategies that resist human immunodeficiency virus escape. Journal of Virology 2005, 79:1645-1654.
20. Applegate $T L$, Birkett DJ, Mclntyre GJ, Jaramillo AB, Symonds G, Murray JM: In silico modeling indicates the development of HIV-1 resistance to multiple shRNA gene therapy differs to standard antiretroviral therapy. Retrovirology 2010, 7:83.

21. Liu, Haasnoot, Berkhout: Design of extended short hairpin RNAs for HIV-1 inhibition. Nucleic Acids Research 2007, 35:5683-5693.

22. Liu, Haasnoot, Brake T, Berkhout, Konstantinova : Inhibition of HIV-1 by multiple siRNAs expressed from a single microRNA polycistron. Nucleic Acids Research 2008, 36:2811-2824.

23. Brake O, Hooft K, Liu Y, Centlivre M, Jasmijn von Eije K, Berkhout B: Lentiviral Vector Design for Multiple shRNA Expression and Durable HIV1 Inhibition. Mol Ther 2008, 16:557-564.

24. Yu JY, Taylor J, DeRuiter SL, Vojtek AB, Turner DL: Simultaneous inhibition of GSK3alpha and GSK3beta using hairpin siRNA expression vectors. Mol Ther 2003, 7:228-236.

25. Schuck S, Manninen A, Honsho M, Fullekrug J, Simons K: Generation of single and double knockdowns in polarized epithelial cells by retrovirus-mediated RNA interference. Proc Natl Acad Sci USA 2004, 101:4912-4917

26. Anderson J, Akkina R: HIV-1 resistance conferred by siRNA cosuppression of CXCR4 and CCR5 coreceptors by a bispecific lentiviral vector. AIDS Res Ther 2005, 2:1.

27. Gonzalez S, Castanotto D, Li H, Olivares S, Jensen MC, Forman SJ, Rossi JJ, Cooper LJ: Amplification of RNAi-targeting HLA mRNAs. Mol Ther 2005, 11:811-818.

28. Saayman, Barichievy, Capovilla, Morris, Arbuthnot, Weinberg, Bowyer: The Efficacy of Generating Three Independent Anti-HIV-1 siRNAs from a Single U6 RNA Pol III-Expressed Long Hairpin RNA. PLOS ONE 2008, 3: e2602.

29. Sano , Li, Nakanishi, Rossi : Expression of Long Anti-HIV-1 Hairpin RNAs for the Generation of Multiple siRNAs: Advantages and Limitations. Mo/ Ther 2008, 16:170-177.

30. Zhu X, Santat LA, Chang MS, Liu J, Zavzavadjian JR, Wall EA, Kivork C, Simon MI, Fraser ID: A versatile approach to multiple gene RNA interference using microRNA-based short hairpin RNAs. BMC Mol Biol 2007, 8:98.

31. Bartel DP: MicroRNAs: genomics, biogenesis, mechanism, and function. Cell 2004, 116:281-297.

32. Kim V: MicroRNA biogenesis: coordinated cropping and dicing. Nat ReV Mol Cell Biol 2005, 6:376-385.

33. Mcintyre GJ: Antiviral shRNA for HIV-1. Ph.D The University of New South Wales (UNSW), The School of Biotechnology and Biomolecular Sciences (BABS); 2006

34. Liu YP, Eije KJv, Schopman NC, Westerink J-T, Brake Ot, Haasnoot J, Berkhout B: Combinatorial RNAi Against HIV-1 Using Extended Short Hairpin RNAs. Molecular Therapy 2010, 17:1712-1723.

35. Gou, Weng, Wang, Wang, Zhang, Gao, Chen, Wang, Liu : A novel approach for the construction of multiple shRNA expression vectors. J Gene Med 2007, 9:751-763.

36. Henry, Vanderwegen, Metselaar, Tilanus, Scholte, Vanderlaan: Simultaneous targeting of HCV replication and viral binding with a single lentiviral vector containing multiple RNA interference expression cassettes. Mol Ther 2006, 14:485-493.

37. Naito Y, Nohtomi K, Onogi T, Uenishi R, Ui-Tei K, Saigo K, Takebe Y: Optimal design and validation of antiviral siRNA for targeting HIV-1. Retrovirology 2007, 4:80.

38. Mcintyre G, Groneman J, Tran A, Applegate T: An Infinitely Expandable Cloning Strategy plus Repeat-Proof PCR for Working with Multiple ShRNA. PLOS ONE 2008, 3:e3827.

39. Coburn G, Cullen B: Potent and specific inhibition of human immunodeficiency virus type 1 replication by RNA interference. J Virol 2002, 76:9225-9231.

40. Jacque J, Triques K, Stevenson M: Modulation of HIV-1 replication by RNA interference. Nature 2002, 418:435-438.

41. Hu W, Myers C, Kilzer J, Pfaff S, Bushman F: Inhibition of retroviral pathogenesis by RNA interference. Curr Biol 2002, 12:1301-1311.

42. Cullen B: Induction of stable RNA interference in mammalian cells. Gene Ther 2005, 13:503-508.

43. Giering JC, Grimm D, Storm TA, Kay MA: Expression of shRNA From a Tissue-specific pol II Promoter Is an Effective and Safe RNAi Therapeutic. Mol Ther 2008, 16:1630-1636. 
44. Liu YP, Haasnoot J, Ter Brake O, Berkhout B, Konstantinova P: Inhibition of HIV-1 by multiple siRNAs expressed from a single microRNA polycistron. Nucleic Acids Research 2008, 36:2811-2824.

45. McIntyre G, Yu Y, Tran A, Jaramillo A, Arndt A, Millington M, Boyd M, Elliott F, Shen S, Murray J, Applegate T: Cassette deletion in multiple shRNA lentiviral vectors for HIV-1 and its impact on treatment success. Virol J 2009, 6:184.

46. Berkhout B: RNA interference as an antiviral approach: targeting HIV-1. Curr Opin Mol Ther 2004, 6:141-145.

47. Lee NS, Rossi JJ: Control of HIV-1 replication by RNA interference. Virus Res 2004, 102:53-58.

48. Haasnoot, Westerhout, Berkhout : RNA interference against viruses: strike and counterstrike. Nat Biotechnol 2007, 25:1435-1443.

49. Leonard JN, Schaffer DV: Computational design of antiviral RNA interference strategies that resist human immunodeficiency virus escape. J Virol 2005, 79:1645-1654.

50. Mclntyre GJ, Fanning GC: Design and cloning strategies for constructing shRNA expression vectors. BMC Biotechnol 2006, 6:1.

doi:10.1186/1742-6405-8-1

Cite this article as: Mcintyre et al: Multiple shRNA combinations for near-complete coverage of all HIV-1 strains. AIDS Research and Therapy 2011 8:1.

\section{Submit your next manuscript to BioMed Central} and take full advantage of:

- Convenient online submission

- Thorough peer review

- No space constraints or color figure charges

- Immediate publication on acceptance

- Inclusion in PubMed, CAS, Scopus and Google Scholar

- Research which is freely available for redistribution

Submit your manuscript at www.biomedcentral.com/submit 